\title{
Protective effects of hydrogen-rich saline against experimental diabetic peripheral neuropathy via activation of the mitochondrial ATP-sensitive potassium channel channels in rats
}

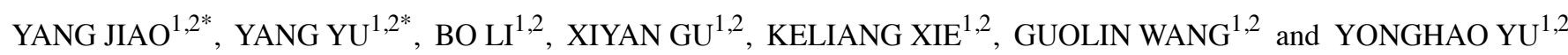 \\ ${ }^{1}$ Department of Anesthesia, Tianjin Medical University General Hospital; \\ ${ }^{2}$ Tianjin Institute of Anesthesiology, Tianjin 300052, P.R. China
}

Received December 18, 2018; Accepted October 14, 2019

DOI: $10.3892 / \mathrm{mmr} .2019 .10795$

\begin{abstract}
It has previously been demonstrated that hyperglycemia-induced oxidative stress and inflammation are closely associated with the development of diabetic complications, including diabetic neuropathy. Additionally, mitochondrial ATP-sensitive potassium (Mito-K-ATP) channels play a homeostatic role on blood glucose regulation in organisms. Molecular hydrogen $\left(\mathrm{H}_{2}\right)$ exhibits anti-inflammatory, anti-antioxidative and anti-apoptotic properties and can be used to treat more than 71 diseases safely. In addition, the diabetes animal models which are set up using streptozotocin (STZ) injection, is a type of high long-term stability, low animal mortality rate and security method. The aim of the current study was to assess the value of hydrogen-rich saline (HS) in diabetic peripheral neuropathy (DPN) treatment and to determine its associated mechanisms in STZ-induced diabetic experimental rats. Additionally, the effects of the Mito-K-ATP channels, oxidative stress, inflammatory cytokines and apoptosis on DPN were also evaluated. From week 5 of STZ injections, HS $(2.5,5$ and $10 \mathrm{ml} / \mathrm{kg})$ was injected into the rat abdominal cavity every day for a period of 4 weeks. The results of the current study demonstrated that HS significantly reduced behavioral, biochemical and molecular effects caused by DPN. However, 5-hydroxydecanoate, a selective Mito-K-ATP channels general pathway inhibitor, partially eliminated the therapeutic effect of HS on DPN. These results indicated that the use of HS may be a novel strategy to treat DPN by activating the Mito-K-ATP pathway and reducing oxidative stress, inflammatory cytokines and apoptosis.
\end{abstract}

Correspondence to: Professor Yonghao Yu, Department of Anesthesia, Tianjin Medical University General Hospital, 154 Anshan Road, Tianjin 300052, P.R. China

E-mail: yuyonghao@126.com

${ }^{*}$ Contributed equally

Key words: diabetic peripheral neuropathy, hydrogen-rich saline, mitochondrial ATP-sensitive potassium channels, oxidative stress

\section{Introduction}

Diabetic peripheral neuropathy (DPN) is a common microvascular complication of diabetes mellitus and can cause pain, limb disorders and repeated hospitalization, which can reduce patient quality of life $(1,2)$. Although DPN is common, it is difficult to cure clinically. Medical intervention has improved patient symptoms, but there is no specific neuroprotective agent for DPN treatment $(3,4)$. Therefore, the treatment of DPN remains a challenge for clinicians.

Due to the complex pathogenicity of DPN, investigations into novel injury mechanism interventions for its treatment are urgently required (5). Some studies have suggested that long-term hyperglycemia can cause energy metabolism disorders and vascular imbalance $(6,7)$. Additionally, mitochondrial ATP-sensitive potassium (Mito-K-ATP) channels function as the high-fidelity metabolic sensors, which couple the intracellular metabolic state to membrane excitability and play a homeostatic role in blood glucose regulation in organisms (8). Moreover, the opening of Mito-K-ATP channels contributes to the regulation of the mitochondrial function and the protection of organs from damage induced by ischemic preconditioning (9). Moreover, several studies have shown a correlation between dysfunction in the Mito-K-ATP channels gating and insulin secretory disorder, which indicated that diabetes and its complications may be associated with the dysfunction of Mito-K-ATP channels $(8,9)$. In addition, reactive oxygen species (ROS) and inflammatory cytokines also serve different roles at a variety of stages, including in the early and late stages of DPN (7). This is consistent with previous studies that have demonstrated that the inhibition of ROS and inflammation may restore energy metabolism, vascular balance, and improve or reverse neuropathy $(7,10)$.

In previous years, a number of studies have indicated that molecular hydrogen (H2) exhibits anti-inflammatory, anti-antioxidative and anti-apoptotic effects in vivo and in vitro, and can prevent stroke, sepsis, multiple organ dysfunction syndrome, ischemia-reperfusion injury and atherosclerosis $(7,11,12)$. Previous studies have revealed that $\mathrm{H} 2$ serves a therapeutic role in sepsis, sepsis-associated organ damage and lipopolysaccharide-induced acute lung injury by reducing inflammatory mediators in cells 
and tissues, including tumor necrosis factor- $\alpha$ (TNF- $\alpha$ ), interleukin (IL)-10, IL-1 $\beta$ and high mobility group box 1 (HMGB1) (13-16). The use of saline in therapeutic doses of hydrogen [hydrogen-rich saline (HS)] is a technique used to transfer molecular hydrogen from blood to the nucleus and mitochondria of cells $(7,17)$. Therefore, HS may be used as a manageable, safe and portable clinical hydrogen delivery method. Based on the results of the aforementioned studies, the present study aimed to indicate that the use of HS may be a novel strategy to treat DPN by activating the Mito-K-ATP pathway and reducing oxidative stress, inflammatory cytokines and apoptosis in diabetic rats.

\section{Materials and methods}

Animals. Adult male Sprague Dawley rats $(n=80)$ were purchased from the Laboratory Animal Center of the Beijing Academy of Military Medical Sciences. Each rat weighed $240 \pm 20 \mathrm{~g}$. A period of 1 week prior to the experiment, all animals were housed in room temperature $\left(20-22^{\circ} \mathrm{C}\right)$ and $30-70 \%$ humidity (5 rats per cage) for 12 -h light and dark cycles fed with normal food and water ad libitum. All experiments in the present study were approved by the Animal Experimental Ethics Committee of Tianjin Medical University General Hospital (license no. 2014-X6-09). The efforts were made to minimize the number of animals used in the studies. During the study, humane endpoints were used in accordance with Tianjin Medical University General Hospital standard operating protocol. In case of infection at the surgical site, wound dehiscence, weight loss $(>20 \%)$ or if the animal became cachectic, had difficulty eating, drinking or moving around freely, the animal was euthanized by inhalation of carbon dioxide. No animal deaths occurred throughout the experiment.

Drugs and reagents. Streptozotocin (STZ; cat. no. S0130) and 5-hydroxydecanoate (5-HD; cat. no. H135), which is a selective Mito-K-ATP blocker, were purchased from Sigma-Aldrich; Merck KGaA; TNF- $\alpha$ (cat. no. RAB0479), IL-6 (cat. no. RAB0311) and IL-10 (cat. no. RAB0247) ELISA kits were purchased from Sigma-Aldrich; Merck KGaA. The HMGB1 (cat. no. BOS-14703) ELISA kit was bought from BOSK (www.bosk-bio.com). A caspase-3 activity kit (cat. no. 5723S) was purchased from Cell Signaling Technology, Inc. The superoxide dismutase (SOD) assay kit (cat. no. 7500-100-K) were purchased from R\&D Systems, Inc. The chloramphenicol acetyltransferase (CAT) assay kit (cat. no. ab238537) and malondialdehyde (MDA) assay kit (cat. no. ab118970) were purchased from Abcam.

Preparation of HS. Previous studies have indicated the details of $\mathrm{HS}$ preparation $(7,18)$. At $0.4 \mathrm{MPa}, \mathrm{H} 2$ was dissolved in normal saline for $6 \mathrm{~h}$, until it reached supersaturation. The saturated HS was sterilized using $\gamma$ rays and preserved in an aluminum bag at an atmospheric pressure of 4 , as described by Ohsawa et al (19). The concentration of hydrogen gas at different time points were detected by a special microelectrode as described in the authors' previous article (7). The HS was replaced weekly to ensure that the hydrogen concentration was maintained at $>0.6 \mathrm{mmol} / 1$, which is the $3 / 4$ of the satura- tion concentration $(0.8 \mathrm{mmol} / \mathrm{l})$ of hydrogen and is enough to produce biological effects $(10,20)$.

Induction of experimental diabetes. A single dose of $55 \mathrm{mg} / \mathrm{kg}$ STZ was dissolved in citrate buffer $(\mathrm{pH} 4.4 ; 0.1 \mathrm{~mol} / \mathrm{l})$ and injected into the abdominal cavity of rats to induce diabetes mellitus. Rats in the control group received an equal amount of citrate buffer. Then, $2 \mathrm{ml}$ of blood for blood sugar determination were collected from the tail vein $48 \mathrm{~h}$ after STZ injection. The main criterion for inclusion in the present study was a blood sugar level of $\geq 13.8 \mathrm{mM}$ (21).

Experimental protocol. In the first experiment, according to the basic record of injury response during week 4 of STZ injection, rats were randomized into the control $(n=8)$, diabetic model (DM; $n=8)$ and HS treatment groups (DM + HS; $n=24)$. From week 4 of STZ injection, the HS group was injected intraperitoneally for 4 weeks $(2.5 \mathrm{ml}, 5 \mathrm{ml}$ and $10 \mathrm{ml} / \mathrm{kg} / \mathrm{day}$, respectively).

Rats in the control group and diabetic model group were intraperitoneally injected with an equal amount of saline. Behavioral tests were performed to assess damage thresholds ( $n=8$ rats per group) at weeks $4,5,6,7$ and 8 . Detection of motor nerve conduction velocity of the sciatic nerve was performed following the last behavioral test. The same groups of rats were used for the mechanical allodynia test and motor nerve conduction velocity test in the present study. The conduction velocity of the sciatic and motor nerves was measured following the last behavioral test. Rats were subsequently decapitated. The MDA content, SOD and CAT activity, and TNF- $\alpha$, IL-6, IL-10 and HMGB1 levels were determined in the serum and sciatic nerve in week 8 . Additionally, the caspase-3 activity in the sciatic nerve was also determined.

In the second experiment, 40 diabetic rats were randomized into 5 groups (8 rats in each group): The control $(n=8)$, diabetic model $(\mathrm{DM} ; \mathrm{n}=8)$, HS treatment $(\mathrm{DM}+\mathrm{HS} ; \mathrm{n}=8)$, diabetic model + 5-HD (DM + 5-HD; $n=8)$ and HS treatment + 5-HD group $(\mathrm{DM}+5-\mathrm{HD}+\mathrm{HS} ; \mathrm{n}=8)$. According to results of the aforementioned experiment, the dose of HS used in experiment two was $10 \mathrm{ml} / \mathrm{kg}$. In the $\mathrm{DM}+\mathrm{HS}$ and $\mathrm{DM}+\mathrm{HS}+5-\mathrm{HD}$ groups, HS (10 ml/kg/day) and 5-HD were intraperitoneally injected daily for 4 weeks starting from week 4 of STZ injection. 5-HD (selective Mito-K-ATP blocker; $20 \mathrm{ml} / \mathrm{kg}$ ) was injected intraperitoneally $30 \mathrm{~min}$ prior to administration.

An intraperitoneal injection of 5-HD (a selective Mito-K-ATP blocker, $20 \mathrm{mg} / \mathrm{kg}$ ) $30 \mathrm{~min}$ prior to administration was required. The activities of SOD and CAT, and the levels of TNF- $\alpha$, IL-6, IL-10 and HMGB1 in the serum and sciatic nerve were determined in week 8. Furthermore, neuronal apoptosis in the sciatic nerve was assessed using a caspase-3 activity kit (Cell Signaling Technology, Inc.).

Assessment of mechanical allodynia. The mechanical threshold of hind paw retraction after mechanical stimulation in rats and the abnormal mechanical pain, was evaluated using an electromechanical pain relief test machine (BME-404) on weeks $4,5,6,7$ and 8 . The rats were placed in a suspension cage on a metal mesh floor for $1 \mathrm{~h}$. A stainless steel wire $(0.6 \mathrm{~mm}$ in diameter) and linear incremental force $(50 \mathrm{~g}$ 
A

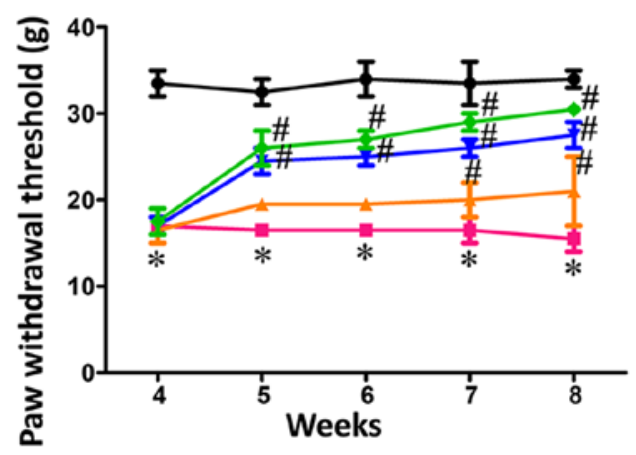

B

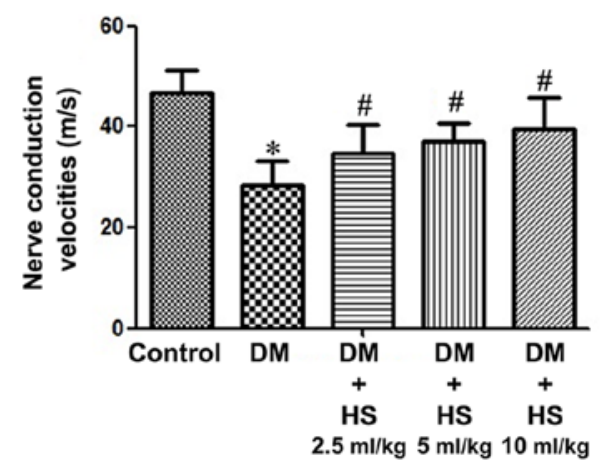

Figure 1. Effects of HS treatment on pain threshold in mechanical hyperalgesia and motor nerve conduction velocity in diabetic rats. On week 4, 5, 6, 7 and 8 following STZ injection, (A) behavioral tests were performed to assess the nociceptive threshold in rats. On week 8 following STZ injection, (B) the motor nerve conduction velocity of the sciatic nerve was also detected. Data are expressed as the mean \pm standard deviation ( $\mathrm{n}=8 / \mathrm{group}$ ). ${ }^{*} \mathrm{P}<0.05 \mathrm{vs}$. the control group; ${ }^{\text {P }}<0.05$ vs. the DM group. STZ, streptozotocin; DM, diabetic model; HS, hydrogen-rich saline.

cut-off) were used to stimulate the bottom of the rear claw to avoid tissue damage. The force $(\mathrm{g})$ of the claw withdrawal was subsequently recorded and this was repeated three times per rat, as described by Fu et al (11).

Motor nerve conduction velocity (MNCV). The nerve conduction velocity was measured at week 8 using a BL-420 biomechanical system (BL-420; Chengdu TaiMeng Co., Ltd.). Rats were anesthetized using pentobarbital $(30 \mathrm{mg} / \mathrm{kg})$ and placed on a heating pad in a greenhouse at $25^{\circ} \mathrm{C}$. The rectal temperature was kept at $37^{\circ} \mathrm{C}$. Proximal nerve stimulation and distal action potential were recorded. The nerve was stimulated using square wave pulses (duration, $0.01 \mathrm{msec}$; intensity, $1 \mathrm{~V})$ transmitted by bipolar recording electrodes. The average values of six potential traces were recorded using electrodes. $\operatorname{MNCV}(\mathrm{m} / \mathrm{s})=($ distance between stimulating electrode and recording electrode)/latency, as described by Xu et al (22).

Sample collection. At the end of 8 weeks of treatment, the animals were anesthetized with $30 \mathrm{mg} / \mathrm{kg}$ of pentobarbital. After a few minutes, the rats were gently clamped on their toes with tweezers, if there were no reactions, the rats were judged to have entered a deep anesthesia state. Then, the rats were executed by cervical dislocation as quickly as possible. When the rats stopped breathing, the head was cut off immediately and a total of $2 \mathrm{ml}$ blood was collected. The serum was acquired following centrifugation at $4^{\circ} \mathrm{C}$ for $15 \mathrm{~min}$ at $3,000 \mathrm{x} \mathrm{g}$ and stored at $-80^{\circ} \mathrm{C}$ until assayed. Moreover, the left sciatic nerves of all rats were fixed with $10 \%$ formalin buffer solution for $24 \mathrm{~h}$ at $4^{\circ} \mathrm{C}$. The right sciatic nerve of all rats was removed immediately, frozen in liquid nitrogen and preserved at $-80^{\circ} \mathrm{C}$. Sciatic nerve samples were homogenized in frozen PBS and centrifuged for $10 \mathrm{~min}$ at a speed of $10,000 \mathrm{x} \mathrm{g}$ at $4^{\circ} \mathrm{C}$. All the samples were long-term preservation in liquid nitrogen prior to testing. Standard commercial kits (Bio-Rad Laboratories, Inc.) were used to determine tissue protein concentrations.

Measurement of MDA, SOD and CAT. The content of MDA and the activity of SOD and CAT in serum and sciatic nerve samples were determined using a commercial kit. All readings were measured using a spectrophotometer at $490 \mathrm{~nm}$ (Beckman 640B).

Measurement of inflammatory cytokines. Serum and sciatic nerve samples were used to detect TNF- $\alpha$, IL-6, IL-10 and HMGB1 levels using specific ELISA kits.

Measurement of apoptosis. The apoptotic neurons evaluation of the sciatic nerve was performed in week 8 . All rats were euthanized following the last behavioral assessment and apoptosis was detected using a Caspase- 3 activity kit.

Statistical analysis. Each experimental was repeated 3 times. All data were reported as the mean \pm standard deviation. An unpaired t-test (if the value exhibited a Gauss distribution) or the Man-Whitney U test (if the value did not exhibit a Gaussian distribution) to analyze the difference between two groups and a one-way analysis of variance with Bonferroni post hoc test to analyze the interactions among all groups. $\mathrm{P}<0.05$ was considered to indicate a statistically significant result and significance tests were two tailed. GraphPad prism software (version 5.0; GraphPad Software, Inc.) and SPSS statistical software (version 16.0; IBM Corp.) was used for statistical analysis.

\section{Results}

Effect of HS treatment on mechanical hyperalgesia and $M N C V$. Compared with the normal control group, the withdrawal threshold of feet in the diabetic group was significantly reduced $(\mathrm{P}<0.05)$ and this was attenuated in the $\mathrm{DM}+\mathrm{HS}$ groups. Compared with the other DM + HS groups, the withdrawal threshold of claws in diabetic rats treated with HS $(10 \mathrm{ml} / \mathrm{kg})$ was significantly decreased $(\mathrm{P}<0.05)$. HS therapy corrected for the reduction of the paw withdrawal threshold, which was caused by diabetes, in a dose-dependent manner (Fig. 1A). MNCV deficiency was also indicated in diabetic rats. However, the nerve conduction velocity in the treatment group was significantly decreased compared with the control group $(\mathrm{P}<0.05)$. HS therapy corrected for the decrease in 

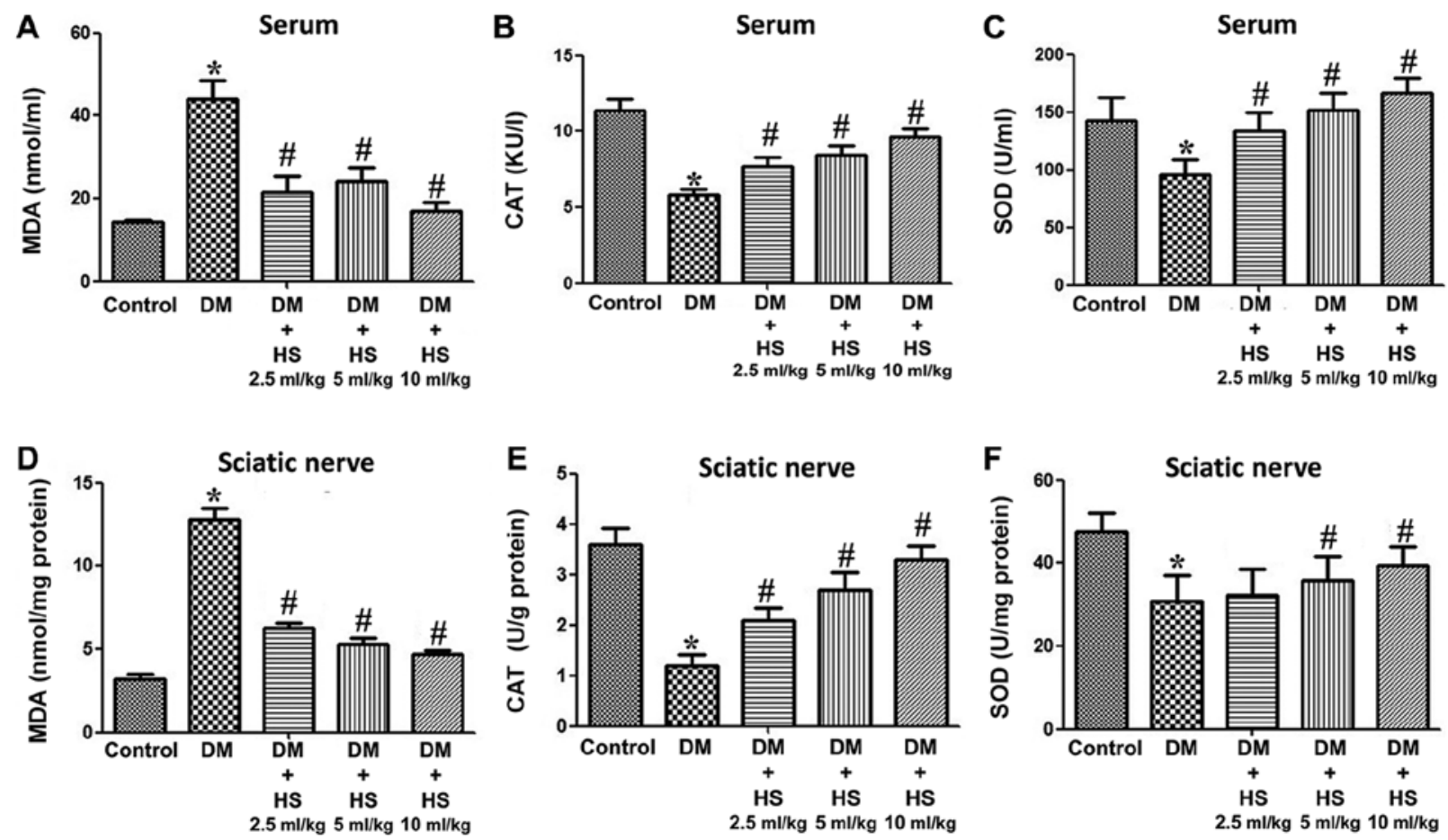

Figure 2. Effects of HS on changes in antioxidant enzymes and oxidative products in the serum and sciatic nerve of diabetic rats. On week 8 following streptozotocin injection, the serum and sciatic nerve were harvested to determine the levels of (A) MDA, (B) CAT and (C) SOD in the serum, and (D) MDA, (E) CAT and (F) SOD in the sciatic nerve. Data are expressed as the mean \pm standard deviation ( $\mathrm{n}=6 /$ group). ${ }^{\mathrm{P}} \mathrm{P}<0.05$ vs. the control group; ${ }^{*} \mathrm{P}<0.05$ vs. the $\mathrm{DM}$ group. MDA, malondialdehyde; CAT, chloramphenicol acetyltransferase; SOD, superoxide dismutase; DM, diabetic model; HS, hydrogen-rich saline.

MNCV, which is caused by diabetes, in a dose-dependent manner (Fig. 1B).

Effect of HS on MDA, SOD and CAT in the sciatic nerve and serum. As presented in Fig. 2, hyperglycemia caused a significant increase in MDA and a decrease in SOD and CAT in the serum and sciatic nerve of diabetic rats at week 8 compared with the control group $(\mathrm{P}<0.05)$. Compared with the DM group, HS treatment led to a significant decrease in MDA and an increase in the activities of SOD and CAT $(\mathrm{P}<0.05)$. DM + HS treatment, at a dose of $10 \mathrm{ml} / \mathrm{kg}$, significantly mitigated the previous effects compared with the other DM + HS groups $(\mathrm{P}<0.05)$. The results indicated that oxidative stress induced by hyperglycemia could increase the content of MDA in the serum and sciatic nerve, and decrease the activity of SOD and CAT. HS treatment caused a dose-dependent decrease in MDA content and an increase in SOD and CAT activities.

Effect of HS treatment on the levels of TNF- $\alpha, I L-6, I L-10$ and HMGB-1 in the sciatic nerve and serum. As indicated in Fig. 3, TNF- $\alpha$, IL-6, IL-10 and HMGB1 levels were also measured in the serum and sciatic nerve. TNF- $\alpha$ and IL-6 are 'early' inflammatory factors, HMGB1 is a type of 'late' inflammatory factor and IL-10 is an anti-inflammatory factor. The results showed that the levels of TNF- $\alpha$, IL-6, Il-10 and HMGB1 in serum and sciatic nerve of diabetic rats increased at week $8(\mathrm{P}<0.05$; Fig. 3). However, the levels of TNF- $\alpha$, IL- 6 and HMGB1 in the DM+HS groups $(2.5 \mathrm{ml} / \mathrm{kg}, 5 \mathrm{ml} / \mathrm{kg}$ and $10 \mathrm{ml} / \mathrm{kg} \mathrm{HS}$ ) were significantly decreased compared with those in the DM groups $(\mathrm{P}<0.05)$, but the IL-10 levels in the DM + HS groups were significantly increased compared with the DM group $(\mathrm{P}<0.05)$. The results demonstrated that hyperglycemia increased the pro-inflammatory and anti-inflammatory cytokines in the serum and sciatic nerve Additionally, hyperglycemia decreased pro-inflammatory but not anti-inflammatory cytokines with dose-dependent HS treatment.

Effect of HS treatment on caspase-3 activity. As presented in Fig. 4, the caspase-3 activity of the sciatic nerve was measured at week 8 . The activity of caspase- 3 in the sciatic nerve of diabetic rats significantly increased in the DM group compared with the control $(\mathrm{P}<0.05)$ and HS significantly decreased this activity in the sciatic nerve in a dose-dependent manner $(\mathrm{P}<0.05)$. The results indicated that HS reduced the apoptosis of sciatic nerve cells by reducing caspase- 3 activity in experimental patients with diabetes mellitus.

Mito-K-ATP channels. The role of the Mito-K-ATP pathway in HS neuroprotection was also assessed in the current study. Compared with the diabetic group, the mechanical withdrawal threshold of the hind claw and the sciatic nerve cell pressure in the diabetic + HS group, were significantly increased $(\mathrm{P}<0.05$; Fig. 5). SOD and CAT activity, MDA content and levels of inflammatory cytokines (TNF- $\alpha$, IL-6, IL-10 and HMGB1) in the serum and sciatic nerve were measured at week 8 (Figs. 6 and 7). Compared with the DM group, MDA, pro-inflammatory cytokines (TNF- $\alpha$, IL-6, HMGB1) levels in the DM + HS group decreased significantly, but the IL-10 level, SOD and CAT activity significantly increased $(\mathrm{P}<0.05$; Figs. 6 and 7). In addition to evaluating the apoptosis of the sciatic nerve the data revealed that caspase- 3 activity 

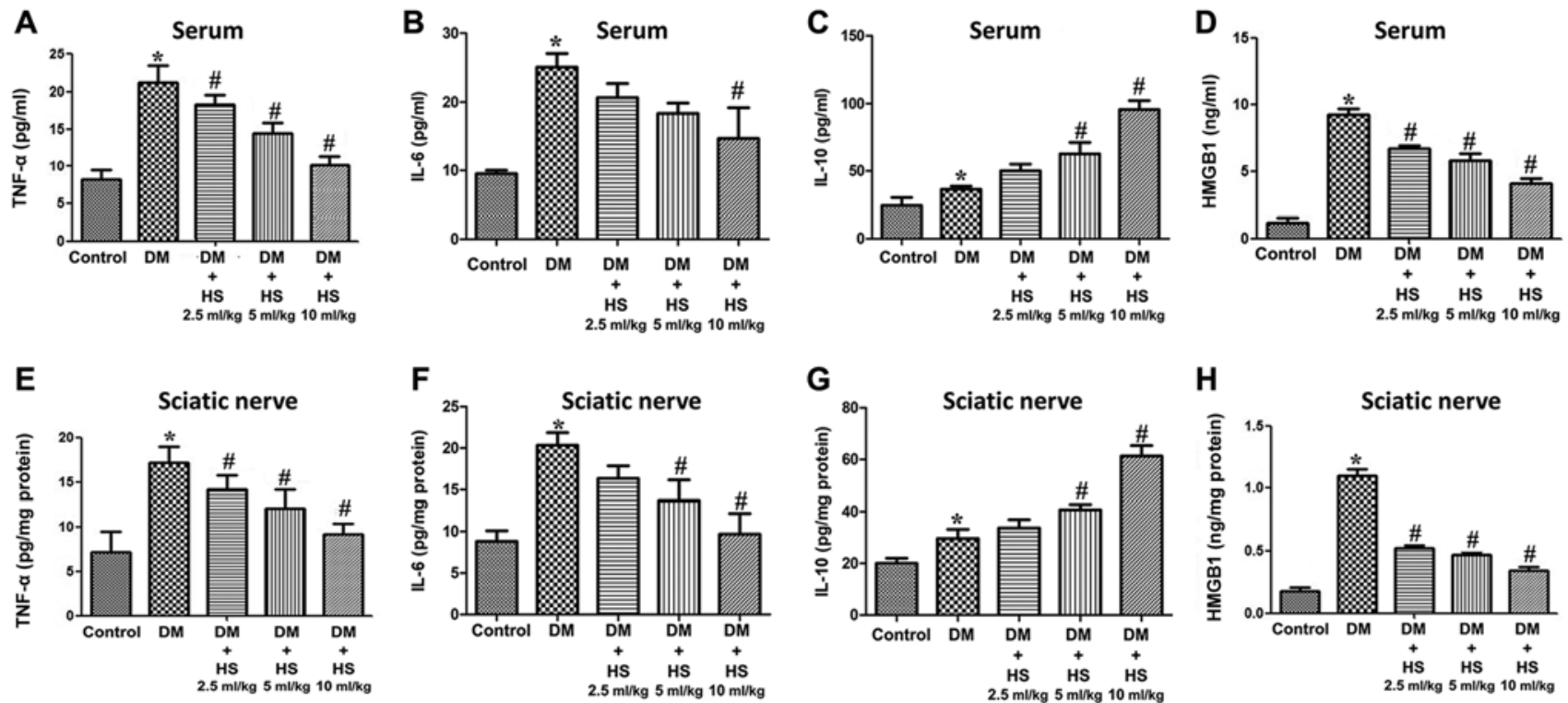

Figure 3. Effects of HS on the levels of pro-inflammatory and anti-inflammatory cytokines in the serum and sciatic nerve of diabetic rats. On week 8 following streptozotocin injection, serum and sciatic nerve were harvested to detect levels of (A) TNF- $\alpha$ in serum, (B) IL-6 in serum, (C) IL-10 in serum and (D) HMGB1 in serum, (E) TNF- $\alpha$ in sciatic nerve, (F) IL-6 in sciatic nerve, (G) IL-10 in sciatic nerve and (H) HMGB1 in sciatic nerve. Data are expressed as the mean \pm standard deviation ( $\mathrm{n}=6 /$ group). ${ }^{\mathrm{P}}<0.05$ vs. the control group; ${ }^{"} \mathrm{P}<0.05$ vs. the DM group. TNF- $\alpha$, tumor necrosis factor- $\alpha$; HMGB1, high mobility group box 1; DM, diabetic model; IL-interleukin; HS, hydrogen-rich saline.

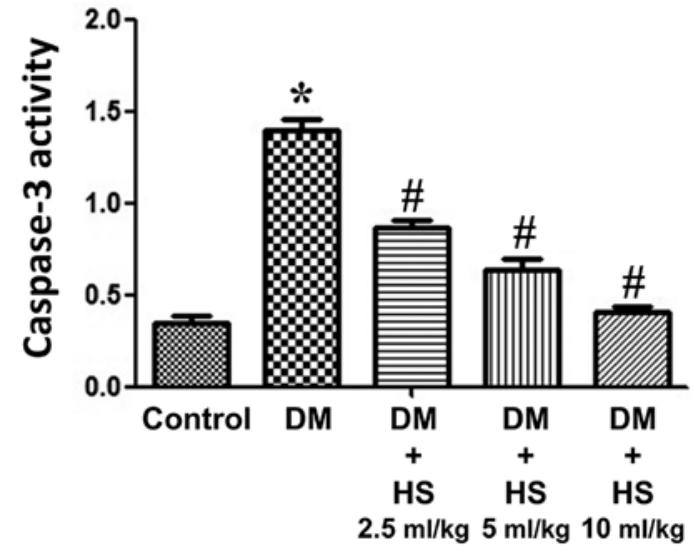

Figure 4. Effects of HS on caspase-3 activity in the sciatic nerve of diabetic rats. On week 8 following streptozotocin injection, the sciatic nerve was harvested to detect caspase- 3 activity. Data are expressed as the mean \pm standard deviation ( $\mathrm{n}=6$ /group). ${ }^{*} \mathrm{P}<0.05$ vs. the control group; ${ }^{*} \mathrm{P}<0.05$ vs. the $\mathrm{DM}$ group. DM, diabetic model; HS, hydrogen-rich saline.

in the DM group was significantly increased compared with the control group $(\mathrm{P}<0.05)$. HS treatment significantly reduced the activity of $\mathrm{c}$ aspase- 3 in the sciatic nerve of diabetic rats $(\mathrm{P}<0.05$; Fig. 8). However, the $\mathrm{DM}+5$-HD and $\mathrm{DM}+5-\mathrm{HD}+\mathrm{HS}$ groups exhibited no significant effect on rat behavior ( $\mathrm{P}>0.05$; Fig. 5), SOD, CAT and MDA levels (P>0.05; Fig. 6), TNF- $\alpha$, IL-6, IL-10 and HMGB1 levels ( $\mathrm{P}>0.05$; Fig. 7 ) and caspase- 3 activity $(\mathrm{P}>0.05$; Fig. 8$)$. The results demonstrated that 5-HD is a selective Mito-K-ATP channels blocker and that the therapeutic effect of HS on STZ-induced diabetic rats can be significantly reduced. These outcomes indicated that activation of the Mito-K-ATP pathway may be conducive to the therapeutic effect of HS in STZ-induced diabetic rats.

\section{Discussion}

The results of the current study demonstrated that HS treatment improved the STZ- induced nociceptive threshold (mechanical and thermal hyperalgesia) and MNCV, but reduced neuronal apoptosis in diabetic rats. On week 5 of STZ injection, rats received HS treatment every day for 4 weeks, which produced beneficial effects in a dose-dependent manner and $10 \mathrm{ml} / \mathrm{kg}$ HS treatment exhibited the most beneficial effect.

The results of the current study also demonstrated that 5-HD (the Mito-K-ATP channels blocker) eliminated the neuroprotective effects of HS treatment. Furthermore, the therapeutic effect of HS was indicated to be closely associated with the decrease of oxidative products (MDA) and pro-inflammatory cytokine (TNF- $\alpha$, IL- 6 and HMGB1) levels, the increase of antioxidant enzyme (SOD and CAT) activities and the anti-inflammatory cytokine (IL-10) level in the serum and sciatic nerve. Additionally, the activity of caspase-3 in the sciatic nerve was downregulated by HS therapy. Therefore, HS may alleviate DPN by activating the Mito-K-ATP pathway, reducing oxidative stress, inflammatory cytokines and apoptosis.

STZ-induced DPN in rats is widely used in neurological, biochemical and histopathological studies $(10,21)$. In the current study, significant differences were observed between STZ-injected and non-diabetic rats, suggesting that diabetic rats exhibited mechanical hyperalgesia. These results are consistent with other observations. Previous studies have demonstrated that behavioral changes can be detected after 1 week of STZ injection and the maximum deterioration time of symptoms in STZ-induced diabetic rats is between 4 and 8 weeks (23). Therefore, the final assessment of oxidative stress, inflammatory cytokines and apoptosis was performed 
A

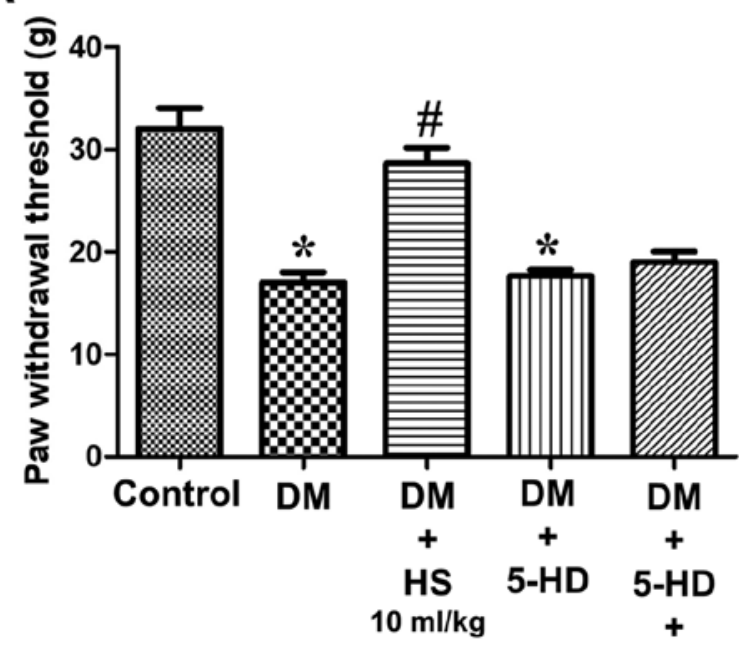

HS
B

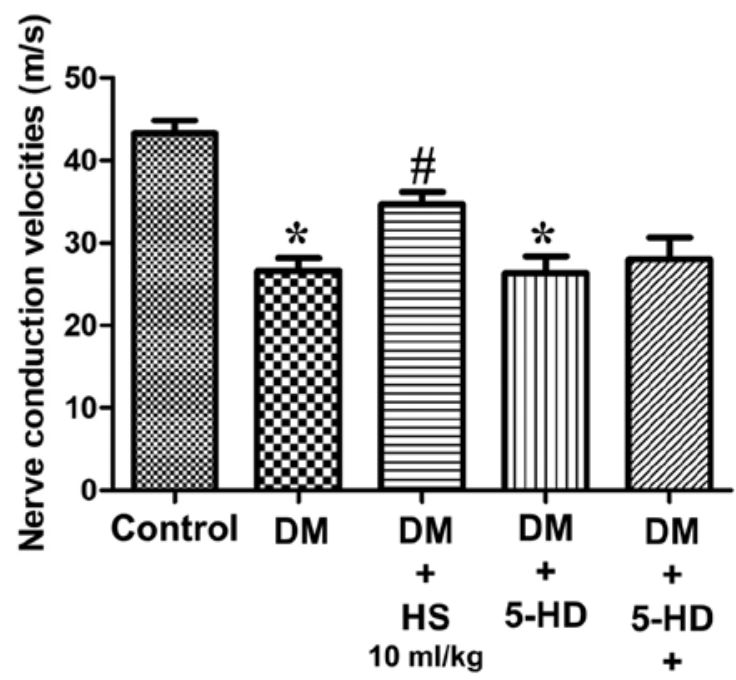

HS

$10 \mathrm{ml} / \mathrm{kg}$

Figure 5. Effects of HS treatment on the pain threshold in mechanical hyperalgesia and motor nerve conduction velocity after blockade of the Mito-K-ATP channels in diabetic rats. In order to inhibit the Mito-K-ATP channels, 5-HD (a selective Mito-K-ATP blocker, $20 \mathrm{mg} / \mathrm{kg}$ ) was injected $30 \mathrm{~min}$ prior to HS administration. On week 8 following streptozotocin injection, (A) pain threshold in mechanical hyperalgesia and (B) motor nerve conduction velocity of sciatic nerve were detected. Data are expressed as the mean \pm standard deviation ( $\mathrm{n}=6 /$ group). ${ }^{~} \mathrm{P}<0.05$ vs. the control group; ${ }^{~} \mathrm{P}<0.05$ vs. the $\mathrm{DM}$ group. HS, hydrogen-rich saline; Mito-K-ATP, mitochondrial ATP-sensitive potassium channels; DM, diabetic model; 5-HD, 5-hydroxydecanoate.

A

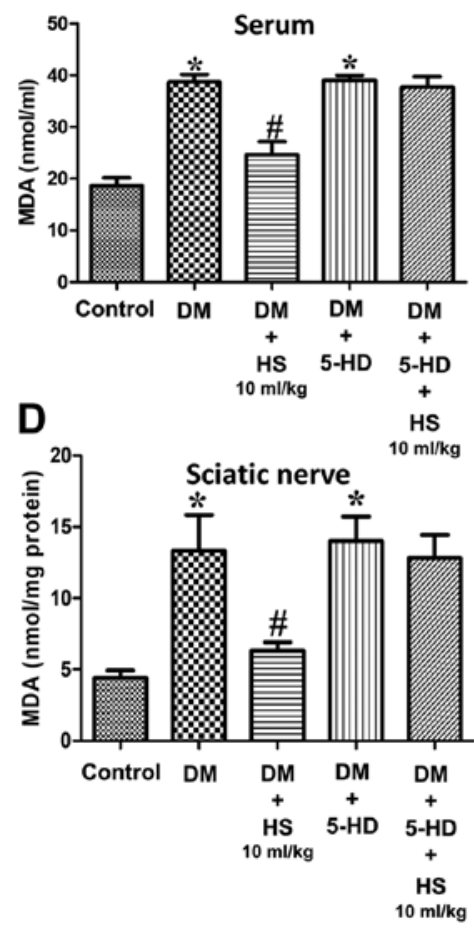

B
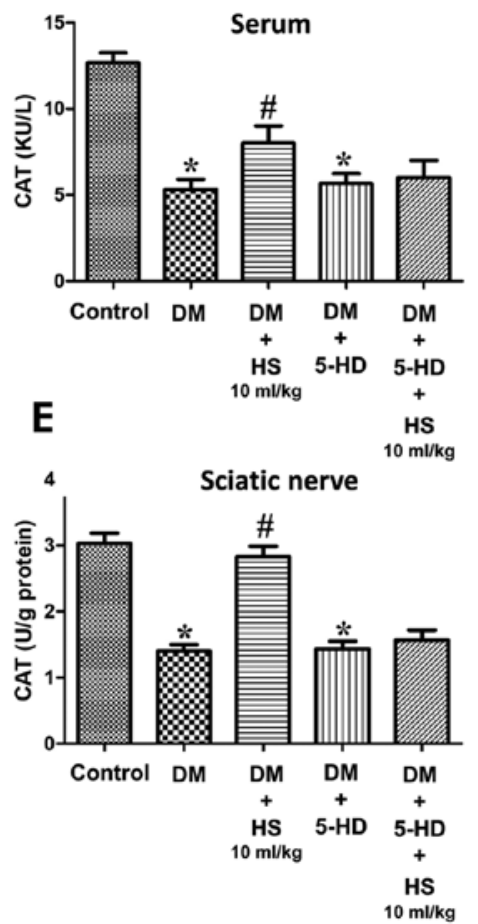
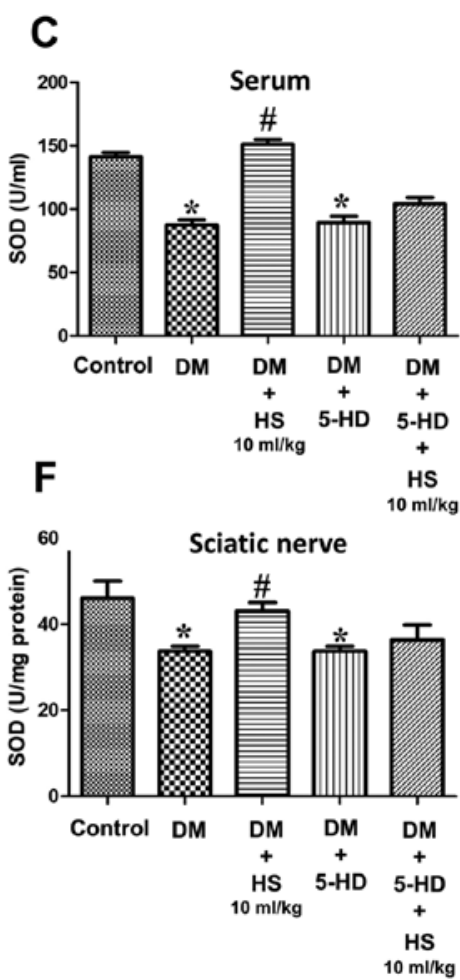

Figure 6. Effects of HS on changes in antioxidant enzymes and oxidative products after blockade of mitochondrial ATP-sensitive potassium channels in the serum and sciatic nerve of diabetic rats. On week 8 following 5-HD and streptozotocin injection, serum and sciatic nerve were harvested to determine the levels of (A) MDA in serum, (B) CAT in serum, (C) SOD in serum, (D) MDA in the sciatic nerve, (E) CAT in the sciatic nerve and (F) SOD in the sciatic nerve. Data are expressed as the mean \pm standard deviation ( $\mathrm{n}=6 /$ group). ${ }^{*} \mathrm{P}<0.05$ vs. the control group; ${ }^{\#} \mathrm{P}<0.05$ vs. the DM group. MDA, malondialdehyde; $\mathrm{CAT}$, chloramphenicol acetyltransferase; SOD, superoxide dismutase; DM, diabetic model; HS, hydrogen-rich saline; 5-HD, 5-hydroxydecanoate.

at week 5 of STZ injection and week 8 of the present study. Behavioral changes were caused by DPN, which is characterized by the mechanical hyperalgesia of large fibers and the sensorimotor deficits of larger fibers. In the present study, HS treatment resulted in a reversal of the lower paw withdrawal threshold and a reduction of MNCV deficiency in 

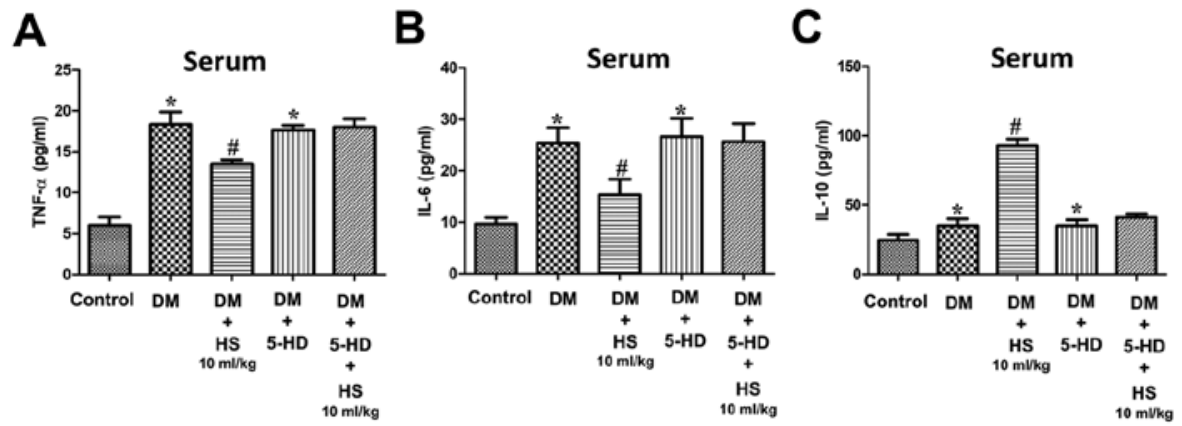

D
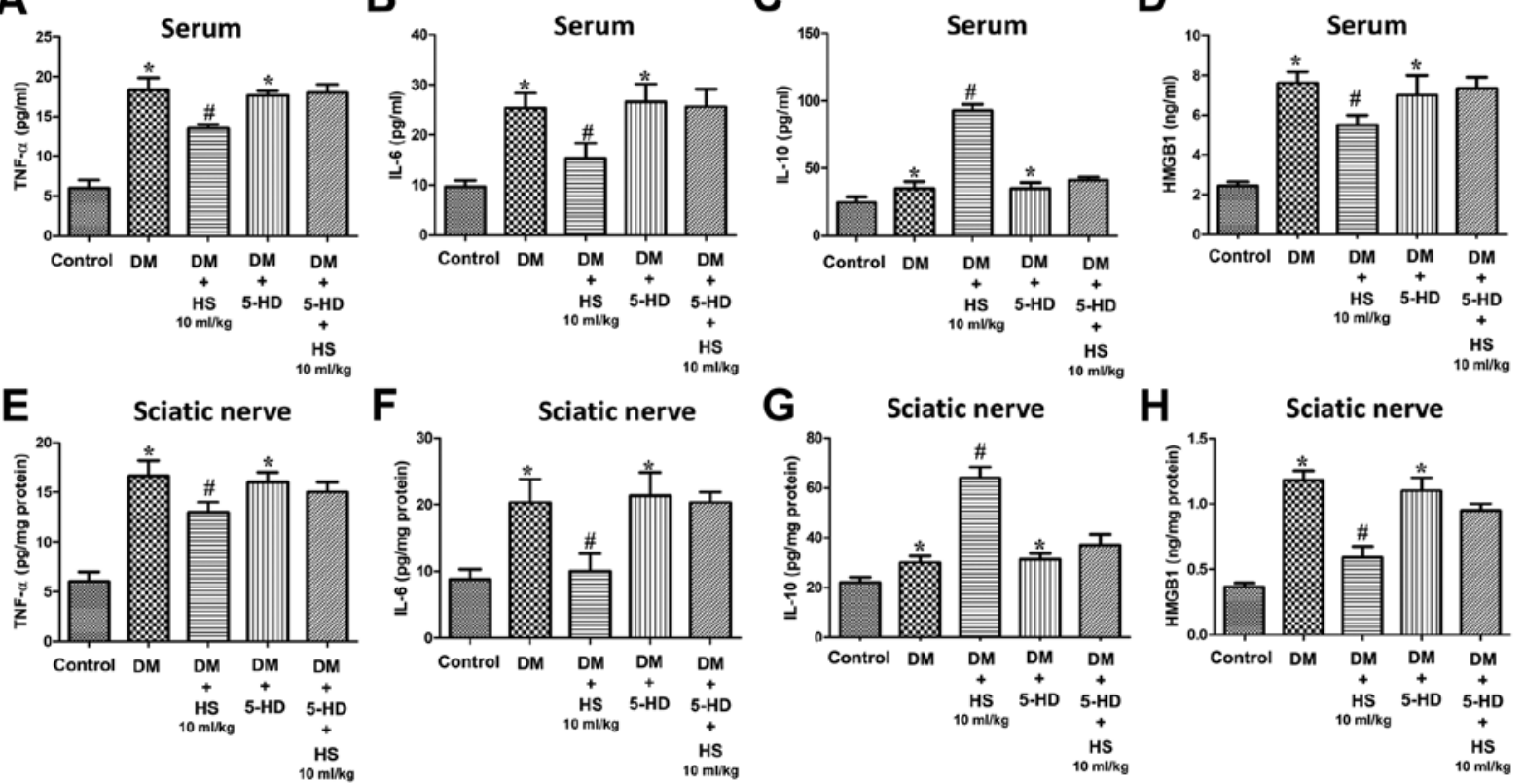

Figure 7. Effects of HS on the levels of pro-inflammatory and anti-inflammatory cytokines after blockade of the mitochondrial ATP-sensitive potassium channels in the serum and sciatic nerve of diabetic rats. On week 8 following 5-HD and streptozotocin injection, the serum and sciatic nerve were harvested to detect the levels of (A) TNF- $\alpha$, (B) IL-6, (C) IL-10 and (D) HMGB1 in the serum and (E) TNF- $\alpha$, (F) IL-6, (G) IL-10 and (H) HMGB1 in the sciatic nerve. Data are expressed as the mean \pm standard deviation ( $\mathrm{n}=6$ /group). ${ }^{*} \mathrm{P}<0.05$ vs. the control group; ${ }^{\prime} \mathrm{P}<0.05$ vs. the DM group. TNF- $\alpha$, tumor necrosis factor- $\alpha$; HMGB1, high mobility group box 1; DM, diabetic model; HS, hydrogen-rich saline; 5-HD, 5-hydroxydecanoate.

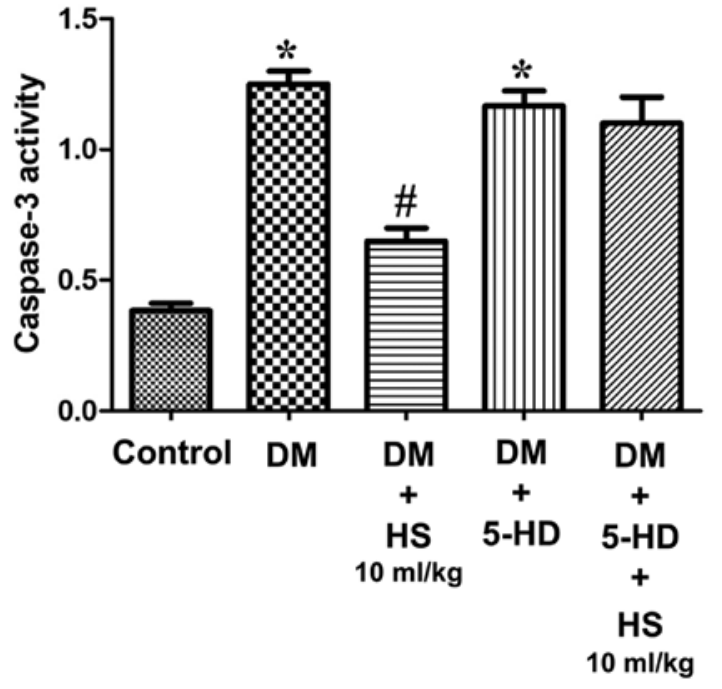

Figure 8. Effects of HS on caspase-3 activity after blockade of the mitochondrial ATP-sensitive potassium channels in the sciatic nerve of diabetic rats. On week 8 following 5-HD and streptozotocin injection, sciatic nerve was harvested to detect caspase-3 activity. Data are expressed as the mean \pm standard deviation ( $\mathrm{n}=6 /$ group). ${ }^{*} \mathrm{P}<0.05$ vs. the control group ${ }^{\text {"P }} \mathrm{P}<0.05$ vs. the DM group. DM, diabetic model; 5-HD, 5-hydroxydecanoate; HS, hydrogen-rich saline.

diabetic rats. Oxidative stress is one of the main causes of the complex pathophysiology of DPN (1,7). Previous studies have indicated that hyperglycemia increases the production of free radicals by increasing intracellular glucose oxidation, which subsequently results in neurotoxicity (7). MDA is a common end point of lipid peroxidation, which is induced by ROS and its concentration is considered to reflect the total level of lipid peroxidation (24). Antioxidant enzymes SOD and CAT counteract the harmful effects of ROS (25). The results of the current study are consistent with previous experimental results that have revealed that the reduction of oxidative damage in the serum and sciatic nerve, and the increase of endogenous antioxidant enzyme activity, may be attributed to the therapeutic effect of HS (7). The quenching of free radicals by HS may be an important mechanism of antioxidant stress and neurotoxicity reduction. In chronic hyperglycemia, the production of endogenous TNF- $\alpha$ accelerates microvascular and neurological problems, and can lead to increased microvascular permeability, hypercoagulability and neurological damage. These neurological problems can promote the development of diabetic microvascular diseases and multiple neuropathies $(4,26)$. The results of the aforementioned study indicated that TNF- $\alpha$ and IL- 6 serve a major role in pain conduction and neurodegeneration (27). HMGB1 can activate ischemic or hypoxic cells to initiate an inflammatory response (28). HMGB1, which is a late cytokine-like mediator,can mediate chronic neuroinflammation and promote progressive neurodegeneration, and serves a key role in diabetic complications (29). In experimental diabetic models, early and late pro-inflammatory cytokines can interact and promote peripheral nerve injury. The results of the current study revealed that diabetic rats exhibited increased inflammatory cytokine release and HS treatment reduced the production and release of these inflammatory cytokines in a dose-dependent manner. Additionally, apoptosis may be an important mode of neuronal death and neurodegeneration in this experimental diabetes mellitus model. At the molecular level, caspase cascades, including caspase-12 and caspase-3, can activate apoptosis. Caspase- 3 which can be activated 
by an initial caspase is considered to be the most important initiator of caspase cascades and its expression is generally regarded as a reliable indicator of apoptosis (30). In the present study, caspase-3 activity was indicated to increase in the diabetic model rats, but decreased markedly during HS treatment. Mitochondria, the source of ROS and the target of sepsis, are considered to be very important in DPN (31). The opening of Mito-K-ATP channels can reduce ROS production in the mitochondria of the heart, brain and spinal cord, activate ROS scavengers and lead to neuroprotection (8). The activation of the Mito-K-ATP pathway can alleviate diabetic neuropathy by reducing oxidative stress and apoptosis $(8,9)$. 5-HD is considered to be an inhibitor of the Mito-K-ATP channels (7). Currently, 5-HD is used to test the participation of the Mito-K-ATP channels and in the present study, it was demonstrated that the neuroprotection induced by HS is mediated by the Mito-K-ATP pathway activation in experimental diabetic models.

The current study has several limitations. First, a small number of time points were measured and the changes in inflammatory cytokines, the activities of CAT, MDA and caspase-3 (and the expression of caspase-3) were only recorded at week 8 . Changes in the serum and/or sciatic nerve at different time points should be assessed in future studies. Second, knockout mice were not used in the present study. However, the inhibitor of Mito-K-ATP (5-HD) that was used in the research can also be used for this purpose. Finally, HS was not added to the control group. However, the authors' previous studies have already reported that there were no statistical differences between control and control plus HS group in different animal models $(12,32,33)$.

In conclusion, the results of the current study demonstrated that HS was able to reduce oxidative stress, the release of pro-inflammatory cytokines and apoptosis by activating the Mito-K-ATP pathway, and may be an effective drug for the treatment of DPN.

\section{Acknowledgements}

Not applicable.

\section{Funding}

The present study was supported by the National Natural Science Fund of China (grant no. 81671888), the Natural Science Foundation of Tianjin (grant no. 18JCYBJC94400), the Science \& Technology Development Fund of Tianjin Education Commission for Higher Education (grant no. 2017KJ194) and the Youth Incubating Fund of Tianjin Medical University General Hospital (grant no. ZYYFY2016036).

\section{Availability of data and materials}

The datasets used or analyzed during the current study are available from the corresponding author on reasonable request.

\section{Authors' contributions}

KX and YoY conceived and designed the study. YJ, BL, YaY, $\mathrm{GW}$ and $\mathrm{XG}$ performed the experiments. YJ and YaY wrote the manuscript. $\mathrm{KX}$ and $\mathrm{GW}$ reviewed and edited the manuscript. All authors read and approved the manuscript.

\section{Ethics approval and consent to participate}

All experiments in the present study were approved by the Animal Experimental Ethics Committee of Tianjin Medical University General Hospital (license no. 2014-X6-09).

\section{Patient consent for publication}

Not applicable.

\section{Competing interests}

The authors declare that they have no competing interests.

\section{References}

1. Chung YC, Lim JH, Oh HM, Kim HW, Kim MY, Kim EN, Kim Y, Chang YS, Kim HW and Park CW: Calcimimetic restores diabetic peripheral neuropathy by ameliorating apoptosis and improving autophagy. Cell Death Dis 9: 1163, 2018.

2. Wang LQ, Chen Z, Zhang K, Liang N, Yang GY, Lai L and Liu JP: Zusanli (ST36) acupoint injection for diabetic peripheral neuropathy: A systematic review of randomized controlled trials. J Altern Complement Med: Sep 26, 2018 (Epub ahead of print).

3. Alam U, Riley DR, Jugdey RS, Azmi S, Rajbhandari S, D'Août K and Malik RA: Diabetic neuropathy and gait: A review. Diabetes Ther 8: 1253-1264, 2017.

4. Vinik AI, Camacho P, Reddy S, Valencia WM, Trence D, Matsumoto AM and Morley JE: Aging, Diabetes, and Falls. Endocr Pract 23: 1117-1139, 2017.

5. Li K, Shi X, Luo M, Inam-U-Llah, Wu P, Zhang M, Zhang C, Li Q, Wang Y and Piao F: Taurine protects against myelin damage of sciatic nerve in diabetic peripheral neuropathy rats by controlling apoptosis of schwann cells via NGF/Akt/GSK3 $\beta$ pathway. Exp Cell Res 383: 111557, 2019.

6. Montero AR, Dubin JS, Sack P and Magee MF: Future technology-enabled care for diabetes and hyperglycemia in the hospital setting. Wold J Diabetes 10: 473-480, 2019.

7. Li Q, Jiao Y, Yu Y, Wang G and Yu Y: Hydrogen-rich medium alleviates high glucose-induced oxidative stress and parthanatos in rat Schwann cells in vitro. Mol Med Rep 19: 338-344, 2019.

8. Liang W, Chen M, Zheng D, Li J, Song M, Zhang W, Feng J and Lan J: The opening of ATP-sensitive K+ channels protects H9c2 cardiac cells against the high glucose-induced injury and inflammation by inhibiting the ROS-TLR4-necroptosis pathway. Cell Physiol Biochem 41: 1020-1034, 2017.

9. Shi N, He J, Guo Q, Liu T and Han J: Liraglutide protects against diabetes mellitus complicated with focal cerebral ischemic injury by activating mitochondrial ATP-sensitive potassium channels. Neuroreport 30: 479-484, 2019.

10. Rojas DR, Tegeder I, Kuner R and Agarwal N: Hypoxia-inducible factor $1 \alpha$ protects peripheral sensory neurons from diabetic peripheral neuropathy by suppressing accumulation of reactive oxygen species. J Mol Med (Berl) 96: 1395-1405, 2018.

11. Fu Y, Ito M, Fujita Y, Ito M, Ichihara M, Masuda A, Suzuki Y, Maesawa S, Kajita Y, Hirayama M, et al: Molecular hydrogen is protective against 6-hydroxydopamine-induced nigrostriatal degeneration in a rat model of Parkinson's disease. Neurosci Lett 453: 81-85, 2009.

12. Li J, Dong Y, Chen H, Han H, Yu Y, Wang G, Zeng Y and Xie K: Protective effects of hydrogen-rich saline in a rat model of permanent focal cerebral ischemia via reducing oxidative stress and inflammatory cytokines. Brain Res 1486: 103-111, 2012.

13. Chen H, Xie K, Chen Y, Wang Y, Wang Y, Lian N, Zhang K and $\mathrm{Yu}$ Y: Nrf2/HO-1 signaling pathway participated in the protection of hydrogen sulfide on neuropathic pain in rats. Int Immunopharmacol 75: 105746, 2019.

14. Wang H, Huo X, Chen H, Li B, Liu J, Ma W, Wang X, Xie K, $\mathrm{Yu}$ Y and Shi K: Hydrogen-rich saline activated autophagy via HIF-1 $\alpha$ pathways in neuropathic pain model. Biomed Res Int 2018: 4670834, 2018. 
15. Xin Y, Liu H, Zhang P, Chang L and Xie K: Molecular hydrogen inhalation attenuates postoperative cognitive impairment in rats. Neuroreport 28: 694-700, 2017.

16. Dong A, Yu Y, Wang Y, Li C, Chen H, Bian Y, Zhang P, Zhao Y, $\mathrm{Yu} \mathrm{Y}$ and $\mathrm{Xie} \mathrm{K}$ : Protective effects of hydrogen gas against sepsis-induced acute lung injury via regulation of mitochondrial function and dynamics. Int Immunopharmacol 65: 366-372, 2018.

17. Hedenstierna G: Mechanisms of postoperative pulmonary dysfunction. Acta Chir Scand Suppl 550: 152-158, 1989.

18. Yang T, Wang L, Sun R, Chen H, Zhang H, Yu Y, Wang Y, Wang G, Yu Y and Xie K: Hydrogen-rich medium ameliorates lipopolysaccharide-induced barrier dysfunction via rhoa-Mdial signaling in Caco-2 Cells. Shock 45: 228-237, 2016

19. Ohsawa I, Ishikawa M, Takahashi K, Watanabe M, Nishimaki K, Yamagata K, Katsura K, Katayama Y, Asoh S and Ohta S: Hydrogen acts as a therapeutic antioxidant by selectively reducing cytotoxic oxygen radicals. Nat Med 13: 688-694, 2007.

20. Chen J, Zhang H, Hu J, Gu Y, Shen Z, Xu L, Jia X, Zhang X and Ding X: Hydrogen-rich saline alleviates kidney fibrosis following AKI and retains klotho expression. Front Pharmacol 8: 499, 2017.

21. Yang C, Gao J, Wu B, Yan N, Li H, Ren Y, Kan Y, Liang J, Jiao Y and Yu Y: Minocycline attenuates the development of diabetic neuropathy by inhibiting spinal cord Notch signaling in rat. Biomed Pharmacother 94: 380-385, 2017.

22. Xu Z,Zhou J, Cai J, Zhu Z, Sun X and Jiang C: Anti-inflammation effects of hydrogen saline in LPS activated macrophages and carrageenan induced paw oedema. J Inflamm (Lond) 9: 2, 2012.

23. Parashar A, Mehta V and Malairaman U: Type 2 diabetes mellitus is associated with social recognition memory deficit and altered dopaminergic neurotransmission in the amygdala. Ann Neurosci 24: 212-220, 2018.

24. Reis R, Charehsaz M, Sipahi H, Ekici AI, Macit C, Akkaya H and Aydın A: Energy drink induced lipid peroxidation and oxidative damage in rat liver and brain when used alone or combined with alcohol. J Food Sci 82: 1037-1043, 2017.

25. Barany T, Simon A, Szabo G, Benkő R, Mezei Z, Molnár L, Becker D, Merkely B, Zima E and Horváth EM: Oxidative stress-related parthanatos of circulating mononuclear leukocytes in heart failure. Oxid Med Cell Longev 2017: 1249614, 2017.
26. Kobayashi M and Zochodne DW: Diabetic neuropathy and the sensory neuron: New aspects of pathogenesis and their treatment implications. J Diabetes Investig 9: 1239-1254, 2018.

27. Sherchan P, Huang L, Wang Y, Akyol O, Tang J and Zhang JH: Recombinant Slit 2 attenuates neuroinflammation after surgical brain injury by inhibiting peripheral immune cell infiltration via Robol-srGAP1 pathway in a rat model. Neurobiol Dis 85 : 164-173, 2016.

28. Xie K, Yu Y, Pei Y, Hou L, Chen S, Xiong L and Wang G: Protective effects of hydrogen gas on murine polymicrobial sepsis via reducing oxidative stress and HMGB1 release. Shock 34: 90-97, 2010.

29. Wang X, Feng C, Qiao Y and Zhao X: Sigma 1 receptor mediated HMGB1 expression in spinal cord is involved in the development of diabetic neuropathic pain. Neurosci Lett 668: 164-168, 2018.

30. Yang X, Yao W, Shi H, Liu H, Li Y, Gao Y, Liu R and Xu L: Paeoniflorin protects Schwann cells against high glucose induced oxidative injury by activating Nrf2/ARE pathway and inhibiting apoptosis. J Ethnopharmacol 185: 361-369, 2016.

31. Roman-Pintos LM, Villegas-Rivera G, Rodriguez-Carrizalez AD, Miranda-Diaz AG and Cardona-Munoz EG: Diabetic polyneuropathy in type 2 diabetes mellitus: Inflammation, oxidative stress, and mitochondrial function. J Diabetes Res 2016: 3425617 , 2016.

32. Meng X, Chen H, Wang G, Yu Y and Xie K: Hydrogen-rich saline attenuates chemotherapy-induced ovarian injury via regulation of oxidative stress. Exp Ther Med 10: 2277-2282, 2015.

33. Liu H, Hua N, Xie K, Zhao T and Yu Y: Hydrogen-rich saline reduces cell death through inhibition of DNA oxidative stress and overactivation of poly (ADP-ribose) polymerase- 1 in retinal ischemia-reperfusion injury. Mol Med Rep 12: 2495-2502, 2015. 